\title{
USING COMMUNITY DATA AND ANALYTICS TO BUILD RESILIENCE TO FLOODING
}

AS THE IMPACTS OF CLIMATE CHANGE WORSEN AND URBANISATION CONTINUES, FLOODING IS BECOMING AN INCREASINGLY SERIOUS PROBLEM FOR COMMUNITIES AROUND THE WORLD. PROFESSOR JOÃO PORTO DE ALBUQUERQUE, AT THE UNIVERSITY OF GLASGOW, UK, IS LEADING THE WATERPROOFING DATA PROJECT, EMPOWERING COMMUNITIES IN BRAZIL AND HELPING THEM BETTER PREDICT AND PREPARE FOR FLOODING EVENTS

\section{TAKK LIKE A DATA ANALST}

DIGITAL LITERACY - a person's ability to use digital platforms to find, evaluate and communicate information

\section{EARLY-WARNING SYSTEM - a system} that warns communities or decision-making organisations about approaching hazards by analysing environmental data and computational models to calculate the likelihood of an event that can cause socio-environmental impacts

FLASH FLOOD - a flood that occurs very rapidly

MARGINAL COMMUNITIES - a group 'on the edge of society' that is less able to be involved in mainstream activities and decisions

RAIN GAUGE - an instrument that collects and measures the amount of rainfall

\section{RISK MODEL - a mathematical} representation that uses data from past events to predict the probability of events happening in the future
Climate change is causing extreme weather events to become more frequent and more severe. This raises serious challenges for many communities, including those vulnerable to flooding. "Extreme rainfall events can be particularly devastating in areas with higher social vulnerability, such as poor neighbourhoods in Brazil," says Professor João Porto de Albuquerque, from the University of Glasgow. He is exploring how to build

communities' resilience to flooding, by engaging them in the process of generating the data used to predict when floods will occur.

João's team is working with communities in two very different Brazilian cities. The first, Rio Branco, is a medium-sized city in the heart of the Amazon region and is vulnerable to river flooding. The second, São Paulo, is Brazil's largest city and has a high level of social inequality. Impoverished neighbourhoods have developed in floodprone areas around São Paulo's edges through unplanned urban growth. The team is working in a neighbourhood called M'Boi Mirim, which frequently suffers from flash flooding during intense rainfall.

In both cities, rapid urbanisation has resulted in the expansion of deprived neighbourhoods, sometimes called 'favelas' or slums, which developed in parallel to official urban planning. Housing in these neighbourhoods is often nondurable and so commonly collapses during flood events. There is a lack of improved water and sanitation infrastructure, and natural drainage through the soil is inhibited by asphalt roads.

In addition, data about the risks and impacts of flooding and other natural hazards are often missing from poor neighbourhoods. "Access to detailed data about a country as huge as Brazil is a big challenge," says João. "Social inequalities are reflected in varying data availability, with data gaps in impoverished marginal urban areas." If there is not sufficient data about rainfall and flooding in an area, then it is very hard to predict when floods may occur. This increases the risk to these communities as they are less likely to have warning before flood events.

COMMUNITY-POWERED DATA

João's team is working closely with communities to generate data to fill these information gaps.

"These data will help us improve risk models, which will in turn improve flood early-warning systems," says João. "We can identify how different areas are impacted by varying levels of rainfall and use 


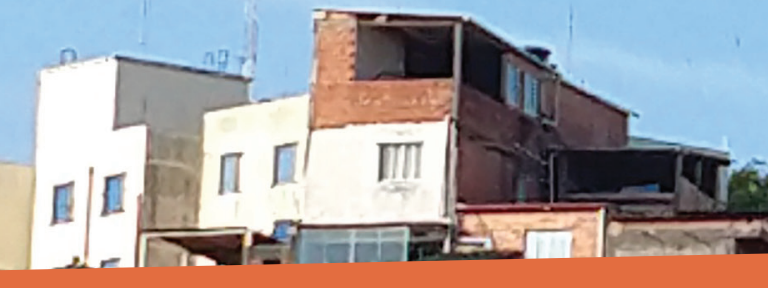

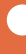 ABOUT URBAN ANALYTICS}

Urban analytics is a new, interdisciplinary field that has emerged in recent years due to the ever-increasing amount of data available about cities. Traditionally, urban data were generated through censuses and surveys. Today, these are supplemented by 'big data', generated through digital technology. For example, satellite imagery can now capture the details of a city to within a few centimetres, while location apps on mobile phones provide data about the movement of people and vehicles in real-time.

\section{Interpreting the huge volumes of data} generated every day, and using them to the benefit of a city and its residents, requires people skilled in urban analytics. "Modern urban data enables a depiction of urban dynamics in much more detail than was possible with traditional data," says João. "An urban analyst must not only have a grounding in urban studies to understand the challenges faced by cities, but also have the skills of a data scientist to process, analyse and visualise urban data. By analysing emerging forms of urban data, we can reimagine cities."

WHAT ARE THE HIGHLIGHTS OF BEING AN URBAN ANALYST?

"What I most enjoy in this interdisciplinary field is that not only do I analyse data and run models on my computer, but I also interact with people in governments, communities and other academic fields to find new approaches to address existing problems," says João. He particularly enjoys doing fieldwork, visiting the communities he is working with and hearing the experiences of people from different cultures and backgrounds. Determining how to connect these experiences to digital technology and data analytics is an enjoyable challenge.

\section{WHAT IS THE FUTURE FOR URBAN ANALYTICS?}

Urban analysts are in the exciting position of advancing this new field, which has the potential to revolutionise how cities are studied and understood. "As digital technologies become more and more widespread, we will have ever-increasing amounts of data about cities," says João, "but also an even bigger challenge to make sense of these data."

Urban analysts will have to develop new methods to analyse huge quantities of data, such as using computational techniques from machine learning and artificial intelligence. But

\section{GXPLORF A GAREER IN URBAN ANALYTIGS}

- As the world's cities continue to expand, urban analysts are needed to address the challenges associated with urbanisation. You could apply your skills in urban analytics to ensure neighbourhoods are socially inclusive, to construct infrastructure that can meet the demands of the population, or to protect communities from disasters.

- The University of Glasgow's Urban Big Data Centre (www.ubdc.ac.uk), where João works, has videos explaining some of the projects they undertake and many useful resources and training materials for people who are interested in using big data to study urban environments.

- A key skill in urban analytics is the ability to visualise and present spatial data, which is commonly done using geographic information systems (GIS). You can watch this video for an introduction to GIS: www.youtube.com/watch?v=659CESxEb5U 


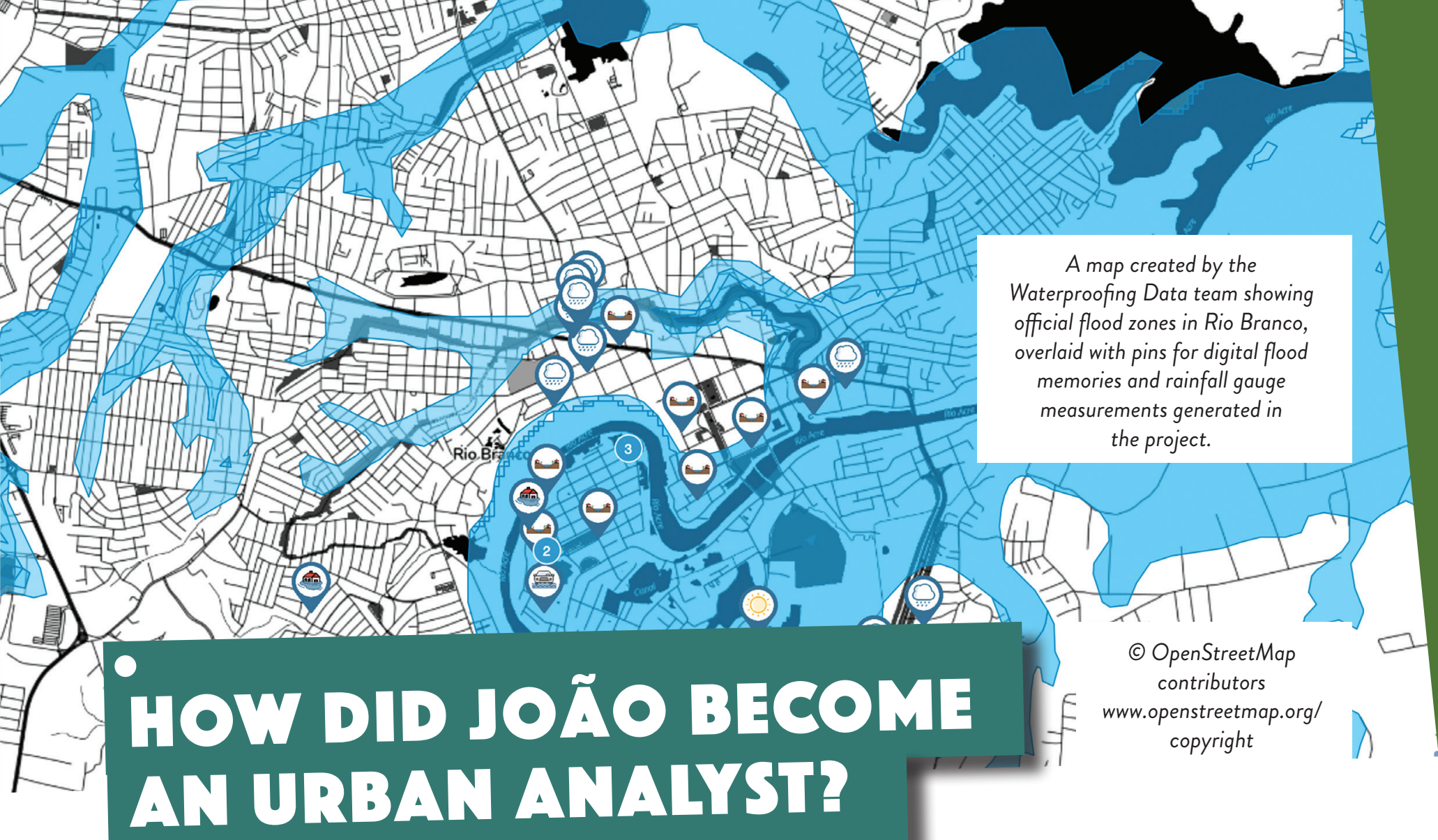

WHAT WERE YOUR INTERESTS WHEN YOU WERE YOUNGER?

I learned programming when I was ten years old and have always been fascinated by computers. I also had a great interest in social justice and environmental issues. Growing up in Brazil, poverty and deforestation of the Amazon Rainforest were very visible to me.

\section{WHAT PATHWAY HAS LED YOU TO} YOUR CURRENT ROLE?

I studied computer science at undergraduate level in Brazil and then completed my PhD in computer science in Brazil and Germany, where I maintained a strong interest in the social usage of technology. When I returned to Brazil, I saw first-hand the catastrophic impacts of flooding in impoverished regions. I found a way to incorporate my computer science expertise into solving social challenges, by getting involved in urban analytics and digital geography. I find this intersection helps match my interests in using data science to address urban and social issues.

WHAT HAVE BEEN YOUR HIGHLIGHTS OF THE WATERPROOFING DATA PROJECT?

This project has enabled me to work with researchers from many different fields, including humanities, social sciences, physical science and engineering. I've found it enriching to learn from so many different perspectives. In addition, we worked directly with government agencies in Brazil and also with the communities on the ground. We learned from their lived experiences of flooding, helping us understand the human dimension behind the data we were collecting.
WHAT ARE YOUR OTHER RESEARCH INTERESTS?

My main interest is investigating new methods to include many diverse voices in the collection and usage of data, to help make cities more sustainable and resilient. In recent years, I have developed a group of projects that focus on empowering vulnerable and deprived communities through citizengenerated data. I've had the privilege of working with partners and communities in countries around the world.

WHAT DO YOU ENJOY OUTSIDE OF WORK?

I enjoy spending time with my wife and three kids. I also occasionally play acoustic guitar and am a big fan of running.

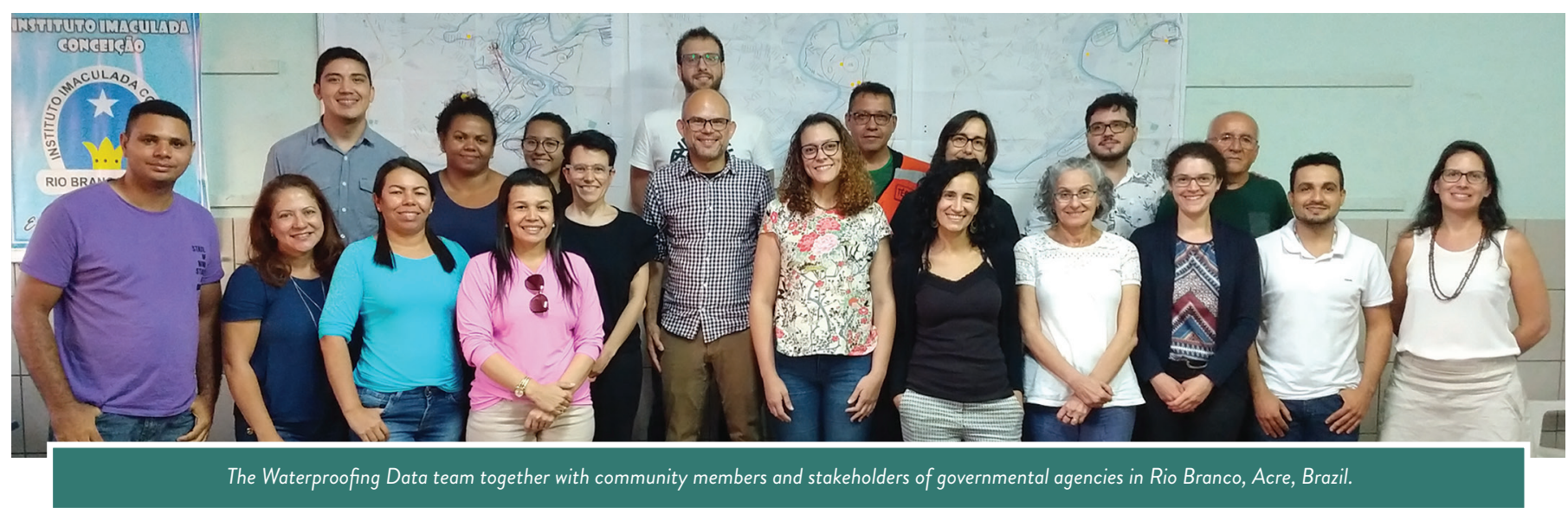

